\title{
Editorial
}

\section{From social media to Monte Carlo}

Journal of Revenue and Pricing Management (2011) 10, 291-292. doi:10.1057/rpm.2011.16

Without doubt, social media (SM) has transformed how we live and how we do things. The world is connected through Twitter and Facebook, and the advancements in mobile platforms means we live in a truly 24hour instant society. As a consequence, distribution channels have changed. In the cities of Tokyo and Seoul, 30 per cent of hotel reservations are on the day of arrival through the mobile phone (Yeoman, 2011). SM refers to a group of internet-based applications that allow the creation and exchange of usergenerated content. SM expedite conversation as opposed to traditional media, which delivers content but does not allow the consumer of that media, whether reader, viewer or listener to participate in the development and dissemination of the content. In response to the rapid adoption of SM, the popular press is replete with articles encouraging hospitality firms to embrace the opportunity that SM provides to listen to, and communicate with, consumers. It appears that the hotel industry is heeding this advice, as evidenced by the volume of hotel companies establishing a presence across the various SM platforms. Noone and colleagues present an excellent article about the debate, effectiveness and how to best leverage SM. Using a quadrant to evaluate the effectiveness of SM on the basis of the information flow and time orientation provides a timely platform for the hotel industry on appropriate revenue management (RM) strategies.

Over the past decade, Sun and colleagues forecast that RM and the cruise line industry have experienced rapid development and the industry is one of the fastest growing in the tourism and leisure sectors. The authors consider a two-stage framework for forecasting for cruise line RM examining pickup methods, most accurate methodology and validity, concluding that despite some similarities in the cruise and hotel industry, the research suggests that the theory and practice of RM forecasting developed for the hotel industry does not directly translate to cruise industry.

Erdelyi and Topalogu developed two methods for making pricing decisions in network revenue management problems. The authors considered a setting where the probability of observing a request for an itinerary depends on the prices and the objective is to dynamically adjust the prices so as to maximize the total expected revenue. The idea behind both of our methods is to decompose the dynamic programming formulation of the pricing problem by the flight legs and to obtain value function approximations by focusing on one flight leg at a time.

Zakhary and colleagues consider the dimensions of forecasting hotel arrivals and occupancy in hotel revenue management systems. In this article, the authors propose a new Monte Carlo simulation approach for the arrivals and occupancy forecasting problem in which yield forecasts densities and parameters are evaluated observing improved results using their method. Karmaker and colleagues focus in accuracy of forecasts in the airline industry proposing a methodology accounting for censorship and correlation of demands. The results show that the opportunity cost of neglecting demand censorship is up to 1 per cent, whereas that for neglecting the dependency of demands can be of the order of 2 per cent.

Kumar and Haider case study analyzes the effectiveness of three types of supply chain 
contracts within the pre-recorded music products supply chain, namely buyback contracts, revenue-sharing contracts and quantity flexibility contracts.

To conclude, Skugge futures article discusses how we think that the champions of tomorrow will move from inside-out (cost-plus) pricing to outside-in (value based) pricing. The challenge with this transition is that revenue managers need to understand customers' preferences and willingness to pay and represent these in a demand function that can be used to optimize the prices. This article lays out a framework for how this can be accomplished.

\section{REFERENCE}

Yeoman, I. (2011) 2050: Tomorrow's Tourism. Bristol, UK: Channelview.

Ian Yeoman Victoria University of Wellington,

New Zealand E-mail: ian.yeoman@vuw.ac.nz 